Brit. J. industr. Med., 1965, 22, 139.

\title{
A SENSITIVE METHOD FOR THE DETERMINATION OF CARBOXYHAEMOGLOBIN IN A FINGER PRICK SAMPLE OF BLOOD
}

\author{
BY \\ B. T. COMMINS and P. J. LAWTHER \\ From the Medical Research Council, Air Pollution Research Unit, \\ St. Bartholomew's Hospital Medical College, London, E.C.1
}

(RECEIVED FOR PUBLICATION FEBRUARY 16, 1965)

\begin{abstract}
About $0.01 \mathrm{ml}$. of blood taken from a finger prick is dissolved in $10 \mathrm{ml}$. of $0.04 \%$ ammonia solution. The solution is divided into two halves, and oxygen is bubbled through one half to convert any carboxyhaemoglobin into oxyhaemoglobin. The spectra of the two halves are then compared in a spectrophotometer, and the difference between them is used to estimate the carboxyhaemoglobin content of the blood either graphically or by calculation from a simple formula. Calibration is simple and need only be done once. A sample of blood can be analysed in about 20 minutes, which includes the time to collect the sample. The method is sensitive enough to be used for the analysis of solutions of blood containing less than $1 \%$ carboxyhaemoglobin.
\end{abstract}

Modern methods for the determination of carbon monoxide in blood have been critically reviewed by Douglas (1962) and by Coburn, Danielson, Blakemore, and Forster (1964), and may be classified as follows: manometric, volumetric, colorimetric, spectroscopic, and those based on the determination of the concentration of carbon monoxide in alveolar air. Only a few of these are sensitive enough to measure low concentrations of carboxyhaemoglobin and most either are time-consuming or require large samples of blood. The methods involving analysis of alveolar air are sensitive but cumbersome.

The method described here has been developed to overcome these disadvantages; it is quick, accurate even at low concentrations of carboxyhaemoglobin, and requires only a minute sample of blood.

\section{Principle of Method}

The basis of the method is that the sample, diluted in $0.04 \%$ ammonia solution, is divided into two parts, from one of which the carbon monoxide is displaced by oxygen by bubbling oxygen through it. The solution containing carboxyhaemoglobin is placed in the sample beam of a spectrophotometer and the oxygenated sample in the reference beam, so that the quantity which the instrument records is the difference between the absorbance of the carboxy- haemoglobin and that of the equivalent amount of oxyhaemoglobin; this is used to determine the concentration of carboxyhaemoglobin in the untreated sample. This difference is measured near its maximum at $420.2 \mathrm{~m} \mu$ and also, in order to define a baseline from which the height of the peak may be measured, at $414 \mathrm{~m} \mu$ and $426 \mathrm{~m} \mu$. The total haemoglobin present is estimated from the difference between the absorbances of an oxygenated sample at $575 \mathrm{~m} \mu$ and $559 \mathrm{~m} \mu$, these being respectively the wavelengths of maximum and minimum absorbance of oxyhaemoglobin.

The absorption spectra of oxyhaemoglobin and carboxyhaemoglobin, and the difference between the corresponding values of the absorbance of the two forms of haemoglobin, are shown in Figure 1. It should be noted that the peak of absorbance of carboxyhaemoglobin occurs at $419.3 \mathrm{~m} \mu$, i.e., at a slightly lower wavelength than the peak of the difference between the absorbances of the two forms. The peaks of the two quantities are, however, sufficiently close together for it to be possible to use measurements at $420 \mathrm{~m} \mu$ in both instances.

\section{Collection of Blood for Analysis}

A 10 ml. specific gravity bottle, i.e., one with a ground glass stopper having a capillary vent, con- 

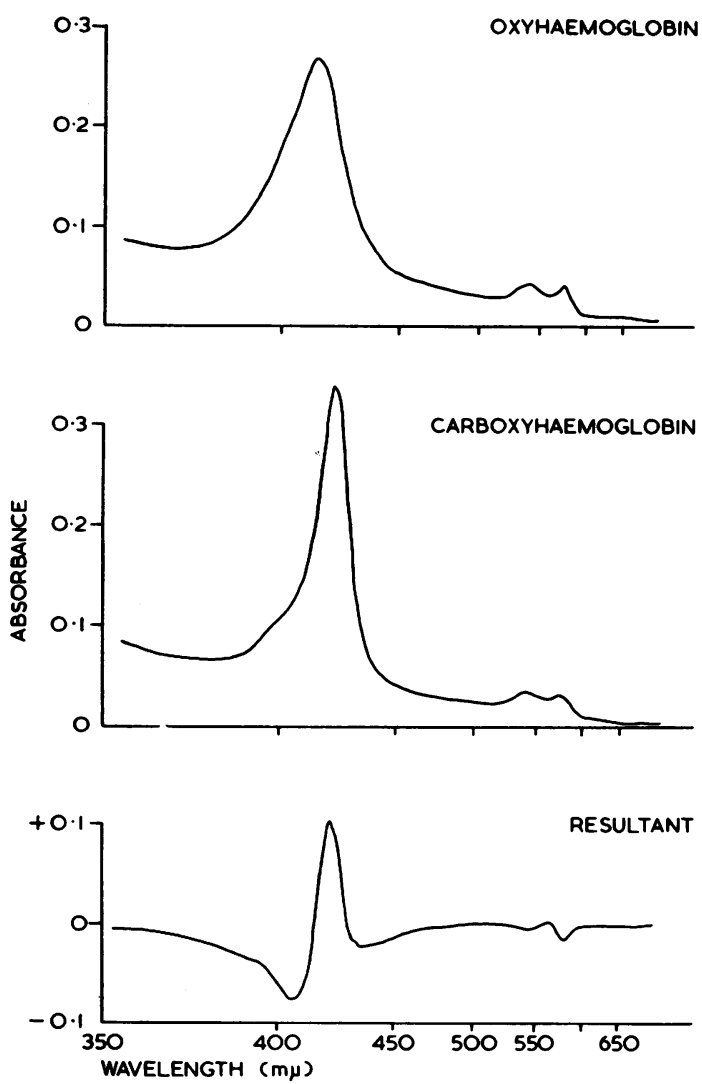

Fig. 1.-Absorption spectra of dilute solution of oxyhaemoglobin, carboxyhaemoglobin, and the difference between the corresponding values of absorbance of the two forms of haemoglobin ('resultant').

taining a small glass bead for mixing, is filled with $0.04 \%$ ammonia solution, and the stopper is inserted so that no air is entrapped. The ammonia solution will contain dissolved oxygen unless it is made up from boiled water and kept in an air-tight bottle, but preliminary experiments showed that the analysis gave the same result in terms of carboxyhaemoglobin whether or not the solution was oxygen-free. Normal distilled water was therefore used in making it up. Blood is sampled from a finger prick either into a capillary tube (approximately $1 \mathrm{~mm}$. bore) or into a $0.01 \mathrm{ml}$. pipette: not more than $0.01 \mathrm{ml}$. of blood should be used normally, and the volume need not be recorded if the carboxyhaemoglobin is to be expressed as a percentage of the total haemoglobin; if, however, the result is to be expressed as volumes of carbon monoxide per $100 \mathrm{ml}$. of blood then the volume of blood must be known. If the blood has been sampled in a capillary tube then about $1.3 \mathrm{~cm}$. of the tube, which contains about
$0.01 \mathrm{ml}$. of blood if the bore is $1 \mathrm{~mm}$., is cut off and dropped into the specific gravity bottle; the stopper is reinserted so that no air is entrapped and the bottle is shaken until the blood has completely dissolved. If a pipette is used the stopper is removed, the tip of the pipette wiped clean and dipped into the solution, and the blood ejected very carefully to ensure that no air is blown into the solution as this could convert some of the carboxyhaemoglobin to the oxygenated form. After the blood has been rinsed out of the pipette the stopper is replaced and again it is imperative to avoid the inclusion of air bubbles. If the sample is not to be analysed immediately, the hole in the stopper is covered with adhesive tape to exclude air. The carboxyhaemoglobin content of samples kept in bottles sealed in this way has been shown to remain unchanged for three days even when the bottles have been transported in a case for part of the time.

The capillary tube method is simpler and more elegant than that using a pipette, but the latter is mentioned since many of the analyses were made on samples collected in this way. If for any reason samples cannot be dissolved immediately, special heparinized capillary tubes, ${ }^{*}$ which prevent the clotting of the blood, can be used. The ends of the tubes may be sealed with plasticine.

\section{Calibration of Spectrophotometer}

To calibrate the method about $0.01 \mathrm{ml}$. of fresh blood is needed. This is dissolved in $10 \mathrm{ml}$. of $0.04 \%$ ammonia as described above, and the absorbance is measured in a $10 \mathrm{~mm}$. cell. If the optimum sensitivity of calibration is to be attained the value of $d$, the difference between the absorbance at $575 \mathrm{~m} \mu$ and that at $559 \mathrm{~m} \mu$, must be kept within fairly narrow limits which are determined by the sensitivity of the spectrophotometer detector: the Beckman DK2 employs a sensitive photomultiplier and with this instrument $d$ may be as high as 0.075 , but when using the Unicam SP 500, $d$ should lie between 0.05 and 0.06 . If necessary the solution should be diluted with ammonia solution, or more blood should be added; until $d$ falls within these limits.

Oxygen from a cylinder is then bubbled through the solution at a rate of about $40 \mathrm{ml}$. per minute for 15 minutes; although inevitably there must be some loss of water vapour by evaporation, this is very small, and the error in analysis which it causes is negligible. The difference $d$ is again measured, and its value should be unchanged unless the original sample contained carboxyhaemoglobin. Part of this

*Hawksley micro haematocrit capillary tubes (heparinized). 
solution $(5 \mathrm{ml}$.) is then put in a test tube and saturated with carbon monoxide by bubbling the gas through it until a slight froth appears, which should be in about 20 seconds if pure $\mathrm{CO}$ is used but will be rather longer if only coal gas is available. Part of the solution is pipetted into a $2 \mathrm{~mm}$. cell and the absorbance is measured at $414 \mathrm{~m} \mu, 420 \mathrm{~m} \mu$, and $426 \mathrm{~m} \mu$. If these are $b, a$, and $c$ respectively, the peak at $420 \mathrm{~m} \mu$ may be regarded as having an 'optical height' $h$ above the mean of the absorbances at $414 \mathrm{~m} \mu$ and $426 \mathrm{~m} \mu$, given by

$$
h=a-(b+c) / 2
$$

Carbon monoxide is again bubbled through the solution, and the measurements are repeated, and if $h$ is unchanged the solution can be regarded as saturated with $\mathrm{CO}$. If a large excess of gas, and in particular of coal gas, is used, $h$ decreases, but the reason for this decrease has not been investigated. (It should be noted that here the term 'optical height' refers to measurements made with a $2 \mathrm{~mm}$. cell, whereas when the symbol is used subsequently it refers to measurements made in a $10 \mathrm{~mm}$. cell unless otherwise specified.)

The two solutions, one saturated with $\mathrm{CO}$ and the other with $\mathrm{O}_{2}$, are put in matched $10 \mathrm{~mm}$. cells and the former is put in the sample beam, the latter in the reference beam, of the spectrophotometer. A tungsten lamp is used as source, and the spectrophotometer slit should be as narrow as the sensitivity of the detector will allow. Most of the work described here was done with a Beckman DK2, with which it was possible to use a slit width of $0.1 \mathrm{~mm}$.: with a Unicam SP500 instrument slit widths of $0.3 \mathrm{~mm}$., $0.2 \mathrm{~mm}$., and $0.1 \mathrm{~mm}$. at wavelengths $414 \mathrm{~m} \mu, 420 \mathrm{~m} \mu$, and $426 \mathrm{~m} \mu$ respectively were found suitable. For each particular wavelength it is essential to use the same slit width throughout, since the absorbance of the solution depends to some extent on the slit width. The difference between the two absorbances is recorded at the same three wavelengths, and $h$ is calculated as above: it should be noted that here $h$ is not the height of the peak of the absorbance of carboxyhaemoglobin but that of the difference between the absorbance of carboxyhaemoglobin and that of the equivalent amount of oxyhaemoglobin. The quantity of haemoglobin present is estimated from the difference $d$ between the absorbances of the oxygenated sample at $575 \mathrm{~m} \mu$ and $559 \mathrm{~m} \mu$. The limiting value of $h$ when the haemoglobin is saturated with carbon monoxide will be designated $h_{0}$ and the limiting value of $d$ when it is completely oxygenated will be designated $d_{0}$.

It has already been mentioned that the value of $d$ must be less than 0.06 for measurements with the Unicam instrument or $\mathbf{0 . 0 7 5}$ for measurements with the Beckman; the reason for this restriction is that, if the solution of blood is too strong, very little light is transmitted through the reference solution, and at low levels of illumination the response of the instrument is non-linear. It must therefore be shown that the relationship between the value of $h$ derived from spectrophotometric measurements and the carboxyhaemoglobin content of the solution is linear as long as $d$ does not exceed the values mentioned above. This was done as follows:

Solutions of oxyhaemoglobin and carboxyhaemoglobin of known composition cannot be prepared by mixing the corresponding proportions of oxygenated and carboxygenated solutions of blood, because the haemoglobin solution saturated with carbon monoxide contains dissolved uncombined gas as well as carboxyhaemoglobin. When, for example, equal volumes of oxy- and carboxyhaemoglobin were mixed there was enough free carbon monoxide to convert all the oxyhaemoglobin, and even when the proportions were six volumes of oxyhaemoglobin solution to one of carboxyhaemoglobin a solution containing about $80 \%$ carboxyhaemoglobin resulted. In the latter case the carboxyhaemoglobin was determined by measuring the 'optical heights' $h$ of the absorbance at $420 \mathrm{~m} \mu$ for the mixed solution, for the $100 \%$ carboxyhaemoglobin solution, and for the $100 \%$ oxyhaemoglobin solution, using $2 \mathrm{~mm}$. cells and with matched cells containing water in the reference beam; the concentration of carboxyhaemoglobin in the mixture was then calculated by proportion. A series of mixtures was then prepared by using the $100 \%$ oxyhaemoglobin solution to dilute the strong carboxyhaemoglobin solution; during the process of dilution great care was taken to avoid oxygenating the carboxyhaemoglobin. For each mixture the difference between the absorbance at $414 \mathrm{~m} \mu, 420 \mathrm{~m} \mu$, and $426 \mathrm{~m} \mu$ and the absorbance of the pure oxyhaemoglobin solution at the same wavelengths was measured, and the 'optical height' of the difference was calculated. The results in Fig. 2 show that within the limits of accuracy of the measurements the response of the instrument was linear. This being so, only one sample is needed for calibration; half the solution is treated with oxygen and the other half is saturated with carbon monoxide.

As mentioned above, the concentration of the haemoglobin solution, as indicated by the value of $d$, must not exceed a critical value which differs from one instrument to another. To determine the critical value for a particular instrument, solutions of oxyhaemoglobin of various concentrations are prepared, and part of each is saturated with carbon monoxide. For each of the original solutions the difference between the absorbances of oxy- and carboxy-forms, and the absorbance of the oxyhaemoglobin, are 


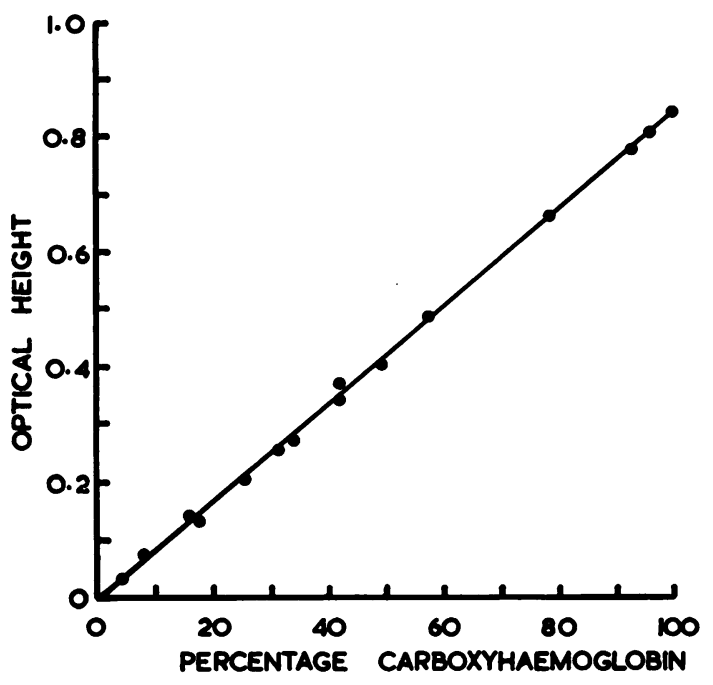

FIG. 2.-Relationship between optical height $h$ derived from measurements at $414 \mathrm{~m} \mu, 420 \mathrm{~m} \mu$, and $426 \mathrm{~m} \mu$ for the Beckman DK2 spectrophotometer and the percentage carboxyhaemoglobin in a diluted blood sample $(d=0.1 ; 10 \mathrm{~mm}$. cells used).

recorded. The 'optical height' $h$ of the difference and the concentration of the haemoglobin in terms of $d$ are calculated, and $h$ is plotted against $d$. At low values of $d$ the points fall on a straight line but above a clearly defined value of $d$ the linear relationship breaks down: the value of $d$ at which this occurs is the critical value for the instrument. If $d$ is higher than this critical value it is still possible to estimate graphically the carboxyhaemoglobin present, using the curved part of this line.

\section{Analysis of Blood}

Part of the solution containing the sample is carefully pipetted into one of a pair of matched $10 \mathrm{~mm}$. spectrophotometer cells, enough being added to ensure that when the ground glass stopper is inserted the cell contains no air. The remainder is poured into a dry $20 \mathrm{ml}$. test tube and oxygen is bubbled into it through a $1 \mathrm{~mm}$. jet for 15 minutes at $40 \mathrm{ml} . / \mathrm{min}$.; this solution is then poured into the other matched cell. The original sample and the oxygenated solution are placed in the sample and reference beams of the spectrophotometer respectively, and the differences between the absorbances of the two solutions at $414 \mathrm{~m} \mu, 420 \mathrm{~m} \mu$, and $426 \mathrm{~m} \mu$ are recorded; $h$ is then calculated.

The absorbance of the oxygenated sample is measured at $575 \mathrm{~m} \mu$ and $559 \mathrm{~m} \mu$ using a $10 \mathrm{~mm}$. cell, and $d$ is calculated. The percentage of haemoglobin present in the carboxy form is then given by:

$$
100 h d_{0} / h_{0} d
$$

where $d_{0}$ and $h_{0}$ are the values obtained during the calibration of the spectrophotometer.

\section{Results}

This method has not been compared with others since we know of none as sensitive using such small volumes of blood. Instead the reproducibility of the method was determined, and for this purpose seven samples were analysed. Samples A and B were freshly taken from members of our laboratory staff; the remaining samples were prepared by mixing stored blood fully saturated with carbon monoxide with normal stored blood. The results are shown in the Table.

It will be noticed that the standard deviation rises steadily with the concentration of carboxyhaemoglobin; the reason for this is not entirely clear. One of the factors contributing to this variation is the random error in the estimation of $d$, but this certainly does not account for the large standard deviation of the results obtained with sample $\mathrm{G}$.

In order to see whether blood samples change in composition whilst they are being taken, $0.1 \mathrm{ml}$. of blood containing carbon monoxide was placed on a glass slide; one $0.01 \mathrm{ml}$. sample was taken immediately and another five minutes later. Both

TABLE

RESULTS OF FIVE CONSECUTIVE DETERMINATIONS OF CARBON MONOXIDE IN SEVEN SAMPLES OF BLOOD

\begin{tabular}{|c|c|c|c|c|c|c|c|}
\hline \multirow{2}{*}{$\begin{array}{l}\text { Estimation } \\
\text { Number }\end{array}$} & \multicolumn{7}{|c|}{ Percentage of Carboxyhaemoglobin in Blood } \\
\hline & $\underset{\mathbf{A}}{\text { Sample }}$ & $\underset{\text { B }}{\text { Sample }}$ & $\underset{C}{\text { Sample }}$ & $\underset{\mathrm{D}}{\text { Sample }}$ & $\underset{E}{\text { Sample }}$ & $\underset{F}{\text { Sample }}$ & $\underset{\mathbf{G}}{\text { Sample }}$ \\
\hline $\begin{array}{l}1 \\
2 \\
3 \\
4 \\
5\end{array}$ & $\begin{array}{l}0.8 \\
0.6 \\
0.6 \\
0.6 \\
0.7\end{array}$ & $\begin{array}{l}1.5 \\
1.4 \\
1.4 \\
1.4 \\
1.4\end{array}$ & $\begin{array}{l}6 \cdot 0 \\
6 \cdot 1 \\
5 \cdot 8 \\
6 \cdot 1 \\
5 \cdot 9\end{array}$ & $\begin{array}{l}9 \cdot 7 \\
9 \cdot 6 \\
9 \cdot 9 \\
9 \cdot 7 \\
9 \cdot 6\end{array}$ & $\begin{array}{l}15.5 \\
15.4 \\
16.1 \\
15.8 \\
15.8\end{array}$ & $\begin{array}{l}20 \cdot 3 \\
20 \cdot 9 \\
20 \cdot 0 \\
20 \cdot 0 \\
20 \cdot 1\end{array}$ & $\begin{array}{l}32 \cdot 3 \\
33 \cdot 1 \\
35 \cdot 7 \\
33 \cdot 7 \\
34 \cdot 5\end{array}$ \\
\hline Mean & 0.66 & 1.42 & 5.98 & $9 \cdot 70$ & $15 \cdot 72$ & $20 \cdot 26$ & $33 \cdot 84$ \\
\hline Standard deviation & 0.07 & 0.05 & $0 \cdot 13$ & $0 \cdot 12$ & $0 \cdot 28$ & 0.38 & 1.34 \\
\hline
\end{tabular}


samples were analysed and found to contain $9.4 \%$ of carboxyhaemoglobin. It can be assumed therefore that negligible loss of carbon monoxide takes place during the short time needed for the collection of the sample.

The authors wish to thank Messrs. G. Gibbs, L.
Hampton, and D. Holland for technical assistance, and Dr. J. McK. Ellison for helpful suggestions with the text.

\section{REFERENCES}

Douglas, T. A. (1962). Ann. occup. Hyg., 5, 211.

Coburn, R. F., Danielson, C. K., Blakemore, W. S., and Forster, R. E. (1964). J. appl. Physiol., 19, 510. 